\title{
Advanced MR imaging of extraventricular supratentorial cortical ependymoma
}

\author{
Sarkar PS ${ }^{1}$, Reddy $R^{2}$, Hoisala $V^{3}$, Hajari $D^{4}$ \\ ${ }^{1}$ Dr Partha Sarathi Sarkar, Senior Resident in Radiology, ${ }^{2}$ Dr Reddy Ravikanth, Post-graduate student in Radiology, \\ ${ }^{3}$ Dr V. Ravi Hoisala, Professor \& Head, Department of Radiology, ${ }^{4}$ Dr Debadatta Hajari, Senior Resident in Medicine, \\ All are affiliated with St. John's Medical College, Bangalore, Karnataka, India.
}

Address for Correspondence: Partha Sarathi Sarkar, Senior Resident in Radiology, St. John's Medical College, Bangalore, Email:drparthasarathisarkar@gmail.com

\begin{abstract}
Ependymomas arise from the ependymal cells of the ventricular system. Ependymomas are relatively uncommon nervous system tumors constituting 2\% - 6\% of all gliomas. They represent $4 \%$ of all CNS tumors in the adult. Extraventricular ependymomas are especially rare with pure cortical ependymomas uncommon. Ependymomas are the most common of the ependymal tumors, and are considered WHO grade II tumors. The location of ependymomas in adults tends to be different than the location of ependymomas in children. Total tumor resection has the best chance of long term survival. The extent of tumor removal continues to be the strongest factor influencing survival and recurrence. MRI of the brain is usually done every 3 months for the first two years following diagnosis to determine the effectiveness of treatment and to watch for possible recurrence. Supratentorial cortical ependymoma without attachment to the ventricular system is a rare tumor in the adult central nervous system. We present a case of a 25 -year-old lady with a large supratentorial cortical ependymoma showing massive calcifications and central cyst formation manifested as headache and generalized seizures.
\end{abstract}

Key word: Ependymoma, Extraventricular, Tumor, Supratentorial, Cortical.

\section{Introduction}

Ependymomas may manifest at any age with no gender predilection. Radiologically, the supratentorial ependymoma is more commonly seated in the brain parenchyma than the infratentorial ependymoma, which is more often located intraventricularly. Schwartz et al reported that $83 \%$ of supratentorial ependymomas are located in the cerebral parenchyma. It is speculated that ependymomas may arise from embryonic rests of ependymal tissue trapped in the developing cerebral hemispheres. With the advent of MRI, the accurate evaluation of the exact relationship between a tumor and the ventricular wall is possible. Recognizing the real ectopic location of an ependymoma is rare. In particular, supratentorial cortical ependymomas without attachment to the ventricular system are extremely rare. We discuss the clinical features and radiological aspects of supratentorial ectopic cortical ependymomas.

Manuscript received $20^{\text {th }}$ April 2016

Reviewed: $4^{\text {th }}$ May 2016

Author Corrected: $16^{\text {th }}$ May 2016

Accepted for Publication $28^{\text {th }}$ May 2016

\section{Case Presentation}

A 25 year old young lady with a 6 month history of chronic headache not relieved with medications presented with vomiting, motor weakness and generalized seizures. On examination, vitals were stable. Neurological examination revealed bilateral papilledema. Routine laboratory investigations were normal. Imaging workup included a CT with contrast enhanced MRI of the brain. MRI showed a large eccentric cortical based solid-cystic lesion in the left frontal lobe with a lobulated solid component appearing hypointense on $\mathrm{T} 1 \mathrm{~W}$ and hyperintense on $\mathrm{T} 2 \mathrm{~W}$ images with heterogenous contrast enhancement. CT showed areas of large chunky calcifications. No intraventricular extension of the lesion was visualized. Single-voxel MR spectroscopy revealed elevated lipid lactate peak and low NAA, choline and creatine peaks in the intralesional region of the tumor. Perilesional MR Spectroscopy findings were normal. Surgical resection 
of the mass lesion was planned with total resection of the tumor undertaken subpially from the surrounding brain parenchyma. Surgical findings suggested no relationship of the lesion with the lateral ventricles. Histopathological examination of the tumor demonstrated round cells with fibrillary cytoplasm, perivascular pseudorosette formation and mitosis with massive calcifications. Immunohistochemistry showed diffuse positive reactivity for glial fibrillary acidic protein (GFAP), vimentin and epithelial membrane antigen (EMA), but not synaptophysin, NF and CD34. These findings were consistent with Ependymoma, WHO Grade 2. Neurosurgeons confirmed gross removal of the tumor. Postoperative course of the patient was uneventful. There was no evidence of residual tumor at postoperative imaging. Patient showed improvement in symptoms and was discharged.
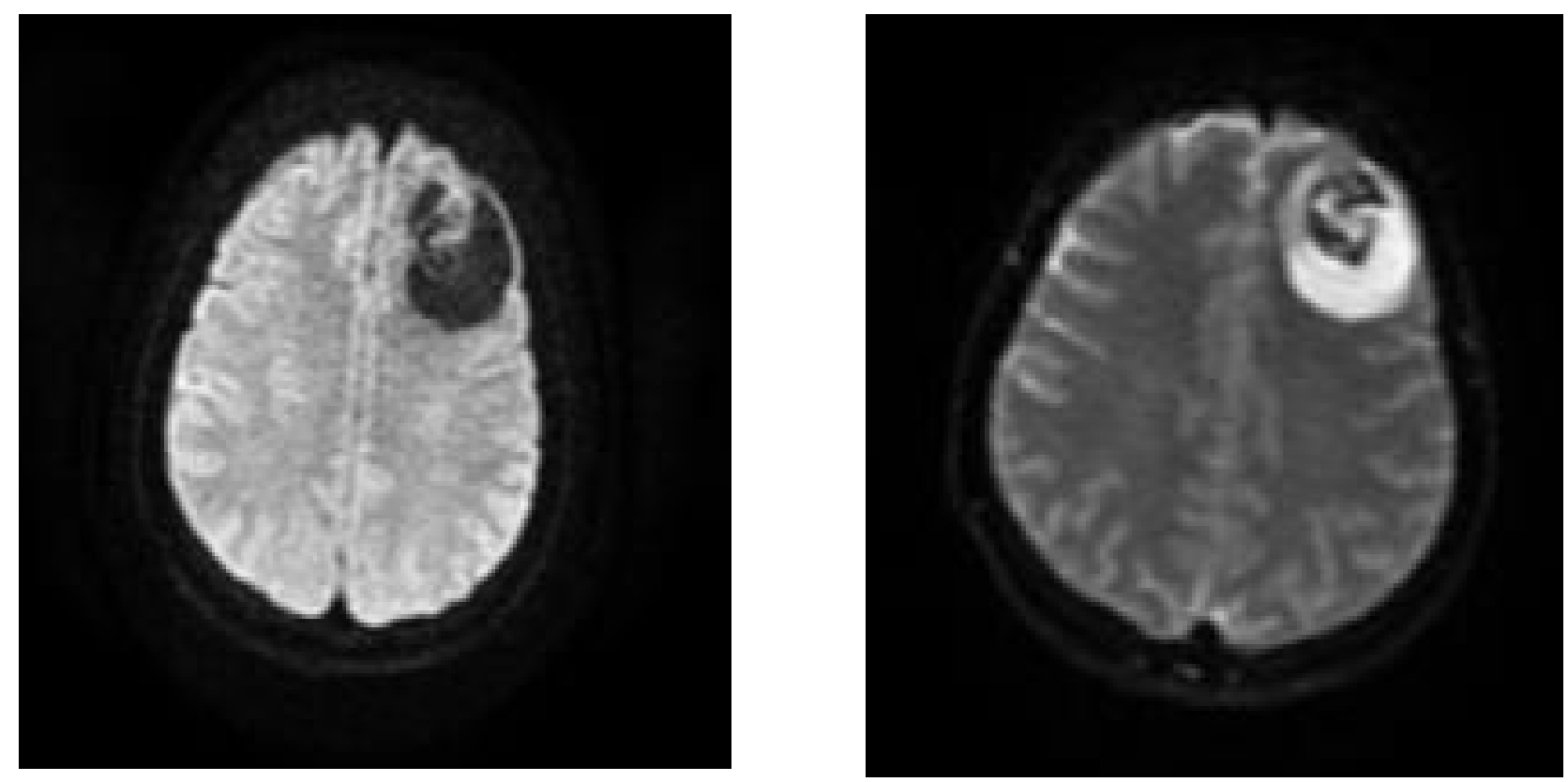

Figures-1 and 2: Axial MR DWI and ADC images showing cortical based lesion in the left frontal lobe with no evidence of diffusion restriction.
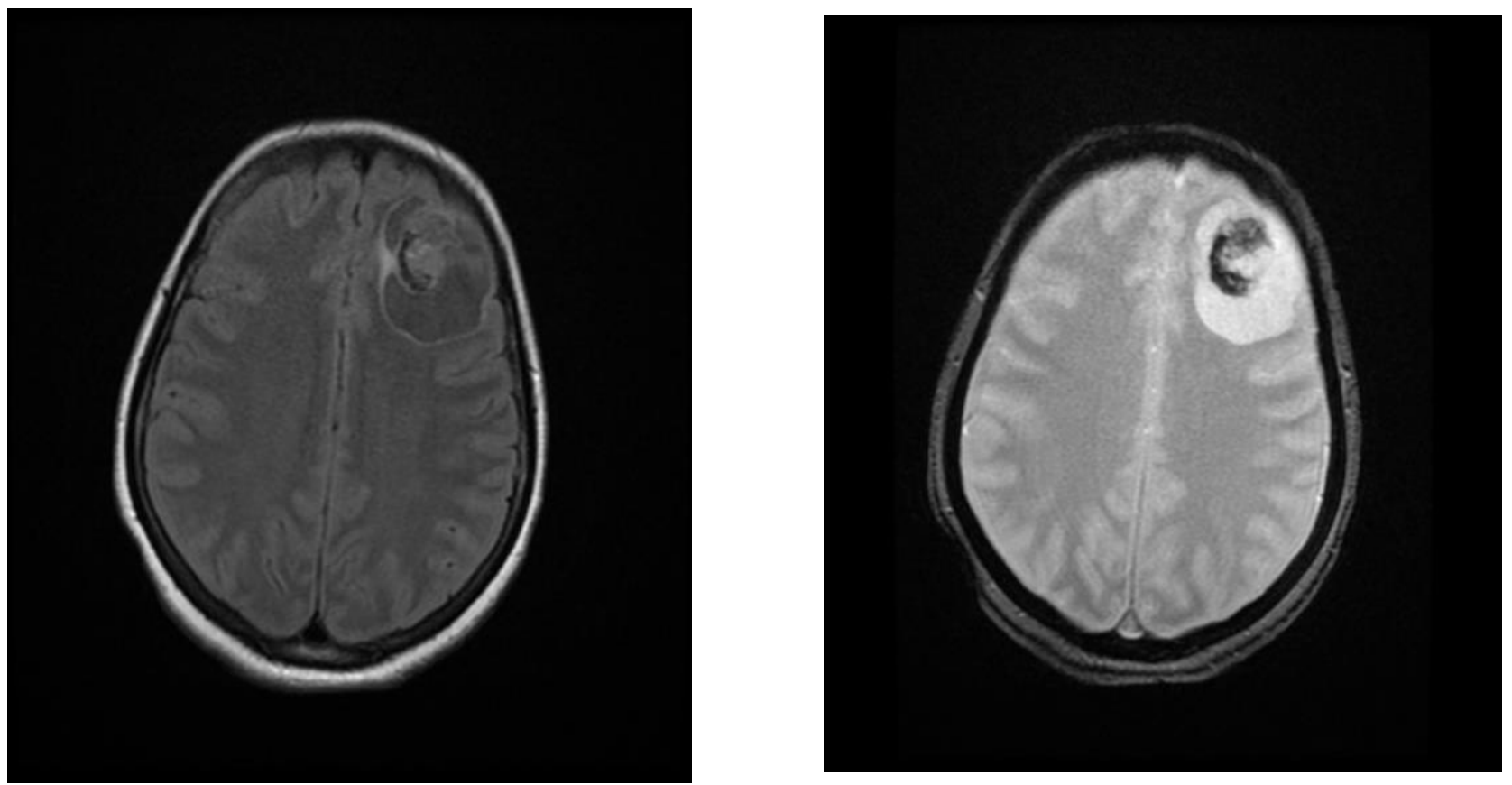

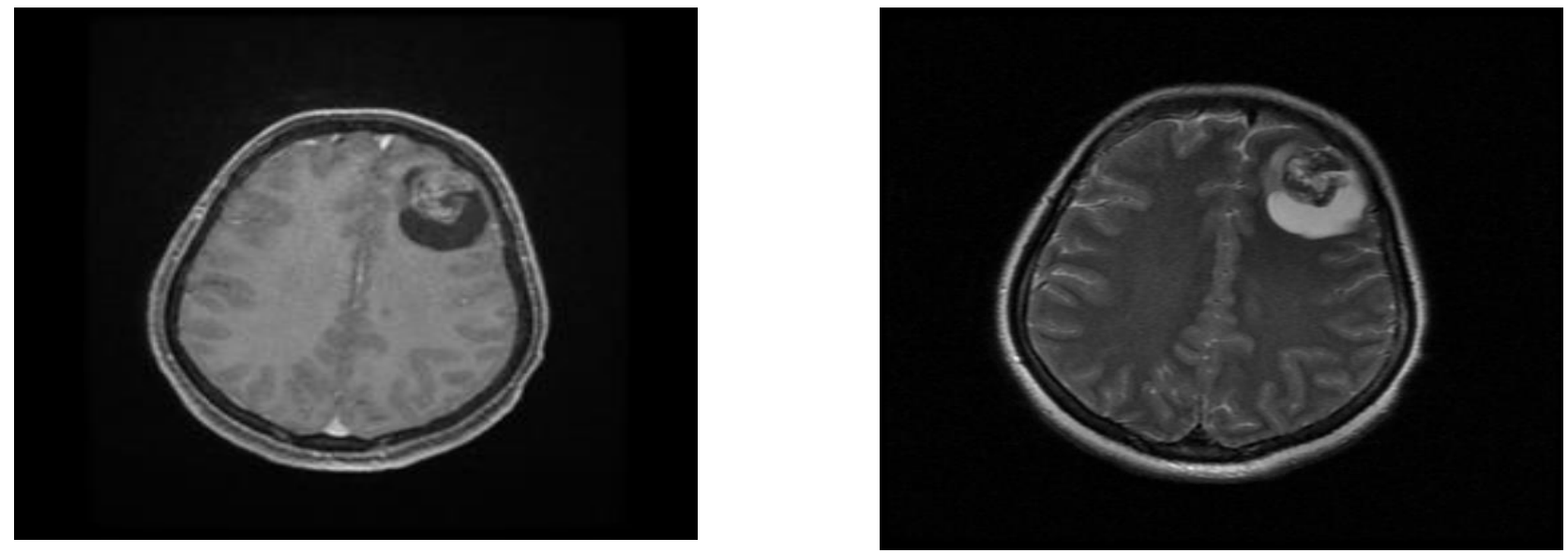

Figures 3, 4, 5 and 6: Axial T2 FLAIR, T2* Gradient recalled ECHO (GRE), T1W+C and T2W images showing signal intensity characteristics of extraventricularsupratentorial cortical ependymoma in the left frontal lobe. Heterogenous contrast enhancement is seen on $\mathrm{T} 1 \mathrm{~W}+\mathrm{C}$ image with blooming of the solid component on $\mathrm{T} 2 *$ Gradient recalled ECHO (GRE) image suggesting calcifications.
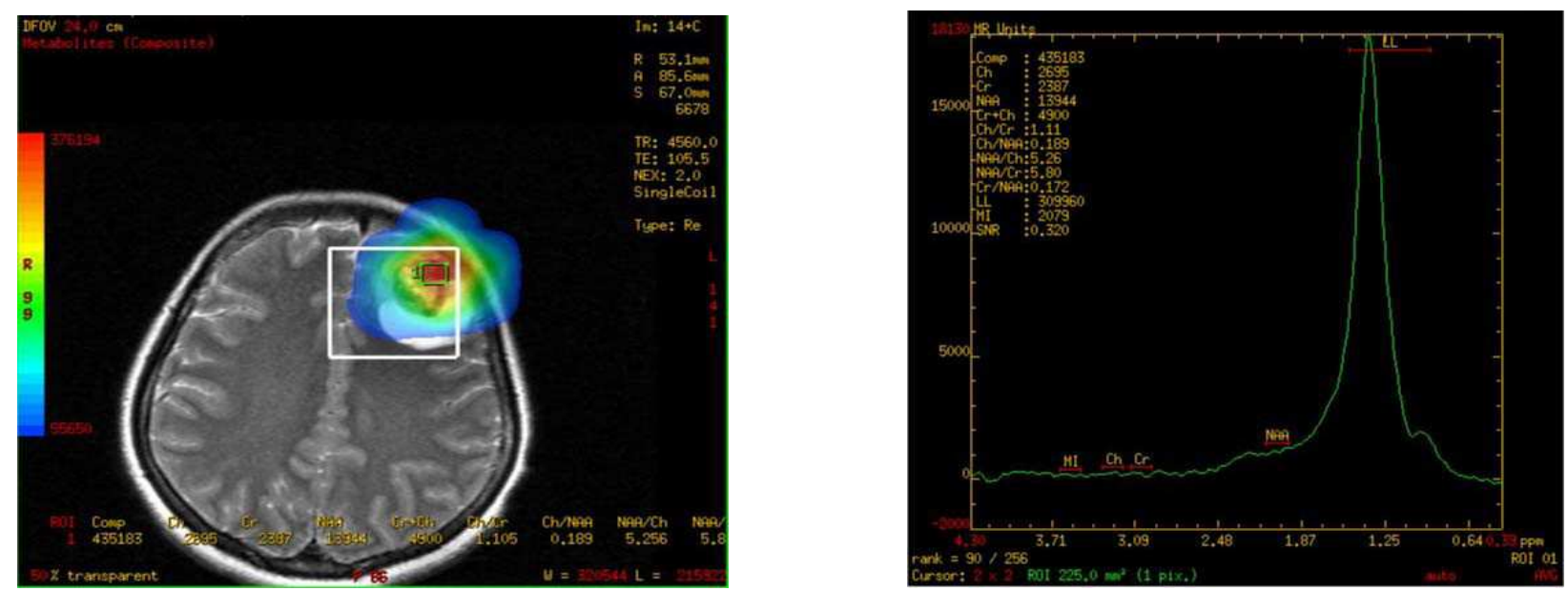

Figures 7 and 8: Coronal T1W and T2 fast spin echo (FSE) images showing the cystic component of the supratentorial cortical lesion and demonstrating no communication with the ventricle.
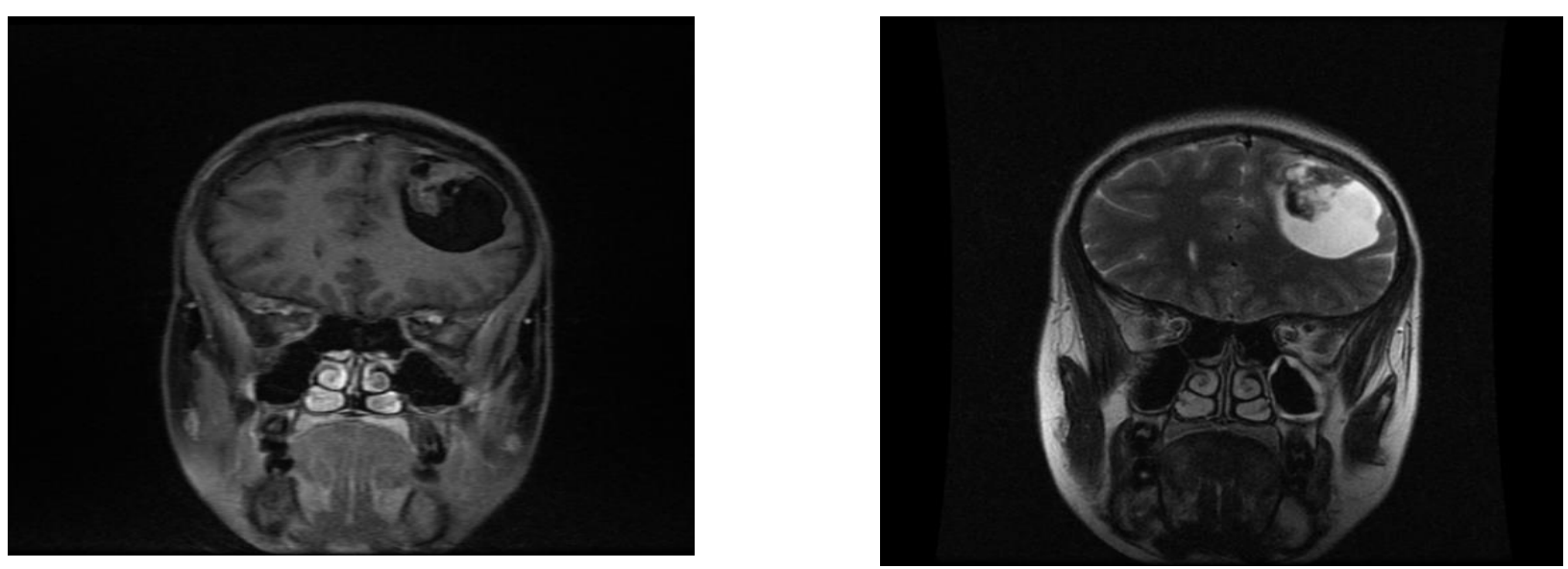

Figures - 9 and 10: Intralesional single-voxel MR spectroscopy images, showing elevated lipid lactate peak, low NAA, choline and creatine peaks. 

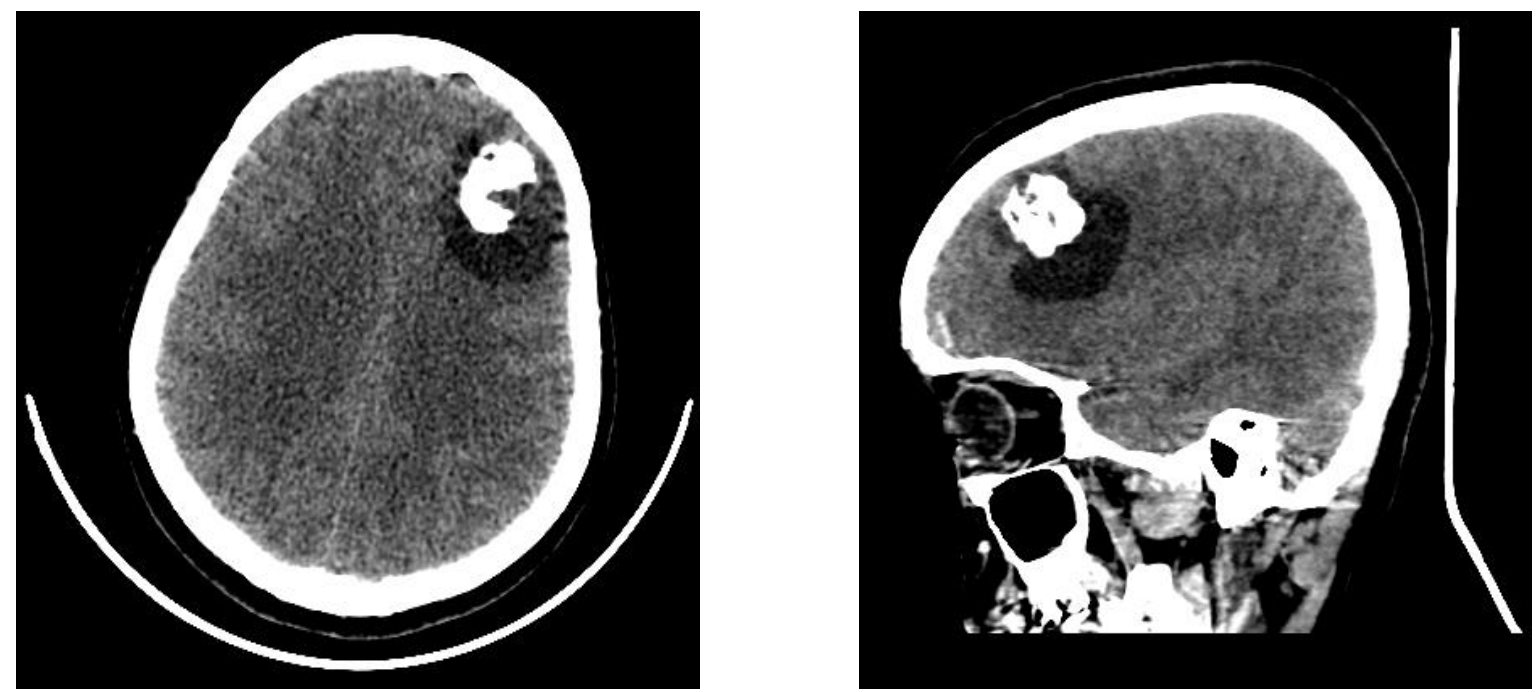

Figure 11 and 12: Axial and sagittal reformatted plain CT images obtained at the time of admission showing cortical solid-cystic lesion with the solid component demonstrating large chunks of calcification.

\section{Discussion}

Ependymomas account for $3-9 \%$ of all neuroepithelialtumors, $6-12 \%$ of paediatric brain tumors, and almost one-third of all brain tumors in patients younger than 3 years. [1] Ependymomas are glial tumors derived from differentiated ependymal cells lining the ventricles of the brain and the central canal of the spinal cord. Ependymomas are considered World Health Organization (WHO) grade II lesions. [2] Posterior fossa ependymoma arises most commonly in children (mean age, 6 years) whereas supratentorial ependymoma generally manifests in an older age group (mean age 18-25 years). Supratentorial ependymomas have a different clinical and radiological presentation than infratentorialependymomas. Patients with supratentorial ependymomas tend to present with focal neurological deficits, headache and seizures. The infratentorialependymomas due to their intraventricular location, will manifest secondary to increased intracranial pressure and hydrocephalus. Supratentorial ependymomas tend to be larger in size than infratentorial ependymomas. Supratentorial lesions often contain a cystic component, while infratentorial lesions are more often solid tumors. Calcifications are common in both (40-80\% of cases).

Minority of ependymomas can arise in the supratentorial parenchyma with no attachment to the ventricular system. Such an ependymoma variant is called an ectopic ependymoma. Extraventricular ependymomas are thought to originate from the rests of ependymal cells that are retained in the brain parenchyma during embryonic development. They are often located near the ventricular margins and may extend into the ventricular system. In comparison to posterior fossa tumors, hydrocephalus is a relatively late presentation, by which time the tumors are typically large, with an average size of over $4 \mathrm{~cm}$ at presentation $[3,4]$.

MRI is the primary imaging modality for the assessment of intracranial ependymomas. MRI avoids the use of ionizing radiation, a desirable strategy in children and young adults who are more prone to radiation induced malignancy than adults. [5] CT is a useful preliminary adjunct, particularly to show tumor calcifications. Radiologically, supratentorial ependymomas are most commonly seated in the brain parenchyma than the infratentorial ependymoma, which is more common located intraventricularly [3,6]. Supratentorial and infratentorial have the same imaging charcateristics which are iso to hypointense relative to normal white matter on unenhanced T1- weighted MR images and hyperintense on T2- and proton-densityweighted MR images. Foci of signal heterogeneity within a solid neoplasm represent methemoglobin, hemosiderin, necrosis, or calcification [7,8]. Ependymomas can display variable contrast enhancement due to necrosis. The differential diagnosis for lesions with the appearance of an extraventricular supratentorial ependymoma should include astrocytoma (both low grade and glioblastoma multiforme), supratentorial primitive neuroectodermal tumor, 
gangliogliom acytoma and oligodendroglioma $[3,7,9]$.

Astroblastomas have to be differentiated from ependymomas. Astroblastomas and ependymomas have a similar histologic appearance characterized by a radiating arrangement of spindle-shaped tumor cells forming perivascular pseudorosettes. These pseudorosettes are similar to those seen in ependymomas and the histopathologic distinction between these two entities is often difficult. The distinct between these two tumors is more obvious radiologically. Astroblastomas are large, peripheral supratentorial tumors. On MRI, these tumors are solidcystic lesions with a characteristic bubbly appearance of the solid component with relatively little associated peritumoral T2 hyperintensity for their large size [10]. Majority of ependymomas are infratentorial and generally centred within or adjacent to the ventricles $[11,12]$. Ependymomas in the supratentorial compartment usually have a cystic component with relatively high signal intensity on T2-weighted images and the solid component lacks the characteristic bubbly appearance seen with astroblastoma [13]. Usually a moderate amount of peritumoral $\mathrm{T} 2$ hyperintensity exists for the their size.

In general, patients with supratentorialependymomas have a better survival rate than patients with posterior fossa ependymomas. The treatment of choice is total radical resection. Postoperative radiation therapy must be administered in every case of partially resected ependymomas as well as for extraventricular ependymomas that are cystic or located near eloquent brain areas, even after apparently total resection. Imaging to assess the extent of resection should be performed less than 48 hours after surgery [14]. Precontrast T1-weighted sequences must always be included to distinguish perioperative haemorrhage from residual enhancing tumor.

\section{Conclusion}

Extraventricular supratentorial ependymoma is an extremely rare tumor, which can rarely present as a single cortical cyst with a solid mural nodule on imaging. The possibility of ependymoma should be considered in the differential diagnosis of a supratentorial cyst with a mural nodule.

Ethical clearance: Obtained from Institutional Ethical Review Board, St. John's Medical College, Bangalore.
Funding: Nil, Conflict of interest: None initiated. Permission from IRB: Yes

\section{References}

1. Afra D, Müller W, Slowik F, Wilcke O, Budka H, Turoczy L. Supratentorial lobar ependymomas: reports on the grading and survival periods in 80 cases, including 46 recurrences. ActaNeurochir (Wien) 1983; 69:243-251.

2. Koeller KK, Sandberg GD. Cerebral intraventricular neoplasms: radiologic-pathologic correlation. Radio Graphics 2002; 22:1473-1505.

3.Armington WG, Osborn AG, Cubberley DA, Harnsberger HR, Boyer R, Naidich TP, Sherry RG (1985) Supratentorial ependymoma: CT appearance. Radiology 157:367-372.

4. Brat DJ, Hirose Y, Cohen KJ, Feuerstein BG, Burger PC. Astroblastoma: clinicopathologic features and chromosomal abnormalities defined by comparative genomic hybridization. Brain Pathol 2000 July :10 (3):342-352.

5. Brody AS, Frush DP, Huda W, Brent RL (2007) Radiation risk to children from computed tomography. Pediatrics 120:677-682.

6. McConachie NS, Worthington BS, Cornford EJ, et al. Review article: computed tomography and magnetic resonance in the diagnosis of intraventricular cerebral masses. Br J Radiol 1994; 67:223-243.

7. Spoto GP, Press GA, Hesselink JR, et al. Intracranial ependymoma and subependymoma: MR manifestations. AJR Am J Roentgenol 1990; 154:837-845.

8. McConachie NS, Worthington BS, CornfordE J,et al. Review article: computed tomography and magnetic resonance in the diagnosis of intraventricular cerebral masses. Br J Radiol 1994; 67:223-243.

9. Swartz JD, Zimmerman RA, Bilaniuk LT. Computed tomography of intracranial ependymomas. Radiology 1982; 143:97-101.

10. Lefton DR, Pinto RS, Martin SW (1998) MRI features of intracranial and spinal ependymomas. PediatrNeurosurg 28:97-105. 
11. Saito T, Oki S, Mikami T, et al. (1999) Supratentorial ectopic ependymoma: a case report. No Shinkei Geka 27:1139-1144.

12. Spoto GP, Press GA, Hesselink JR, Solomon M. Intracranial ependymoma and subependymoma: MR manifestations. AJNR Am J Neuroradiol 1990;11:8391.
13.Furie DM, Provenzale JM. Supra tentorial ependymomas and subependymomas: CT and MR appearance. J Comput Assist Tomogr 1995;19:518-526.

14. Korshunov A, Neben K, Wrobel G, et al. (2003) Gene expression patterns in ependymomas correlate with tumor location, grade, and patient age. Am J Pathol 163:1721-1727.

\section{How to cite this article?}

Sharan R, Singh M, Attri J.P, Singh D. Additive effect of butorphanol in supraclavicular brachial plexus block. Int J Med Res Rev 2016;4 (6):918-923doi: 10.17511/ijmrr.2016.i06.09. 